\title{
Emergence of Chimera-like States in Prefrontal-Cortex Macaque Intracranial Recordings
}

\author{
Evangelos Sigalas*, Junhua $\mathrm{Li}^{\dagger}$, Anastasios Bezerianos ${ }^{\ddagger}$ \\ Singapore Institute of Neurotechnology (SINAPSE), \\ Centre for Life Sciences, National \\ University of Singapore, 117456, Singapore \\ Email: *evsigalas@gmail.com, †juhalee.bcmi@gmail.com, \\ †anastasios.bezerianos@gmail.com
}

\author{
Chris G. Antonopoulos \\ Department of Mathematical Sciences, \\ University of Essex, Wivenhoe Park, CO4 3SQ, UK \\ Email: canton@essex.ac.uk
}

\begin{abstract}
Neural synchronization plays a crucial role in cognitive functions and in performing tasks as it facilitates the transmission of information among the various brain subregions, and thus their communication. In this paper, we use an approach for analyzing and quantifying the emergence of synchronization patterns used previously in the study of data from toy dynamical models, in neurophysiological signals from a macaque monkey and particularly, from prefrontal-cortex intracranial recordings. Specifically, we study the emergence of synchronization patterns in neural ensembles recorded in the macaque brain while the monkey is performing the same delayed saccade task successfully for a number of times. We quantify the emergence of chimeralike states, metastability and coalition entropy in the recordings coming from intracranial arrays implanted in the macaque's brain. Our results show the emergence of spatio-temporal coexisting patterns of synchronized and desynchronized behavior, termed chimera-like states with small metastability during the stage where the target and the distractor appears on the screen and when the go cue appears on the screen for the monkey to report, namely the two most crucial stages of the trials to be termed successful. Finally, we perform a statistical hypothesis test on the calculated quantities over the successful trials and demonstrate that our findings are statistically significant in the sense that they cannot be attributed to randomness.
\end{abstract}

\section{INTRODUCTION}

In the last years, many studies have tried to decode neurophysiological data using information from temporal representations. For example, the authors in [1], decoded the firingrate activity of cortical neurons to predict the 3-dimensional movement of neuroprosthetic devices and in [2], multifractal attributes of electroencephalographic (EEG) recordings were used to predict movement during a motor imagery task.

A well-known approach for decoding neurophysiological data is by utilizing information from the spectral domain. Particularly, McFarland et al. in [3], used the mu and beta rhythms of EEG signals from human brains. In this experiment, the participants managed to successfully move a cursor in a 3-dimensional virtual environment. Further, the authors in [4] used spectral features from electrocorticographic signals (ECoG) of patients with intracranial implanted electrodes to move successfully a cursor on a computer screen.

The use of information from different domains has also been investigated as a means to improve results. In [5], both power spectra features of Local Field Potentials (LFPs) and firingrates of Multi-unit Spikes (MSPs) from the brain of a macaque were used to train the monkey to move a cursor towards a target on a computer screen. In a more ambitious study [6], Event-Related Potentials (ERPs) and EEG spectral features were combined to decode the direction of movement.

Here, we use neurophysiological LFP signals recorded with intracranial implanted electrodes in the brain of a macaque monkey (i.e. prefrontal-cortex intracranial recordings) when performing a delayed saccade task for 240 successful trials [7], and compute the corresponding phase synchronization among the brain subregions with implanted electrode arrays. In this context, a trial is successful if the monkey remembered correctly the location of the target in which case it received food as a reward. We use the chimera-like $\chi$ and metastability $\lambda$ indices, and coalition entropy $H_{c}$ [9] to study the emergence of spatio-temporal phase-synchronization patterns (i.e. chimera-like and metastable states) in the various time windows of the 240 trials. The emergence of spatio-temporal phase synchronization patterns among oscillators where first reported by Kuramoto et al. [10]. In this study, the coexistence of coherence and incoherence in the dynamics of non locally coupled phase oscillators was observed and was later termed chimera states [11].

Since then, there has been increasing interest in chimera states and it has been shown that they are not only limited to phase oscillators, but can also appear in neural systems. Potential application of chimera states in nature include bump states in neural systems and the phenomenon of unihemispheric sleep in birds and dolphins, which sleep with one eye open, meaning that half of the brain is synchronized while the other half being desynchronized. This can be relevant to studies of synchronization in community-based networks.

In [12], the use of a model of phase-coupled oscillators assigned to cortical areas in the cerebral cat cortex was used to investigate the transient and global synchronization. The authors in [13] studied metastable chimera-like states in modular networks inspired by the connectome of the C.Elegans soil worm and in [14], Santos et al., inspired by the cerebral cat cortex, considered a network where the local dynamics is given by Hindmarsh-Rose systems and investigated the emergence 
of chimera-likes states. A remarkable result from this study is the observation of two types of chimera-like states: a spiking chimera-like state with desynchronized spikes, and a bursting chimera-like state with desynchronized bursts. The work in [15] compares the presence of chimera-like states between two different network topologies, an empirical structural network connectivity derived from diffusion-weighted magnetic resonance imaging and a mathematically constructed network with modular fractal connectivity properties.

In this paper, building on these previous works, we seek to study the emergence of spatio-temporal coexisting patterns of synchronized and desynchronized behavior, termed chimeralike states, in neurophysiological signals from a macaque monkey and particularly, from prefrontal-cortex intracranial recordings [7].

\section{EXPERIMENTAL SETUP}

The macaque was trained to perform a delayed saccade task [7]. Electrode arrays of 16- and 32-channels (256 in total), were implanted in the monkey's brain (see fig. 1(b)). The arrays covered the Lateral Prefrontal Cortex and Frontal Eye Fields areas of the monkey's brain.

During the delayed saccade task, the monkey was placed in front of a monitor displaying a grid (see fig. 1(a)). There were 320 trials in total among which 240 successful in the sense that the monkey remembered correctly the location of the target and thus, received food as a reward. Each trial began when the monkey fixated on the fixation point in the center of the grid. The monkey had to remain fixated throughout the trial until the fixation point on the screen disappeared. In fig. 1(a), we present the various time windows in the trials and their corresponding duration. Particularly, a target (red square) was presented for $300 \mathrm{~ms}$ in a random place inside the grid in the monitor, called the Target Stimulus Display (TSD). TSD was followed by a 1 s delay (D1). A distractor (green square) was then presented for 300ms (termed the Distractor Stimulus Display (DSD)), followed by a another 1s delay (D2). After D2, the fixation point disappeared from the monitor which was the go cue for the monkey to report the location of the target using an eye movement. If the monkey remembered correctly the location of the target, it was rewarded with food and the trial was successful. The next trial was then started right after the end of the previous trial.

\section{MATERIALS AND Methods}

The 256 channels are grouped in 8 groups of 32 channels each. The grouping of the channels corresponds to the brain subregions of the macaque as shown in fig. 1(b). Furthermore, the data of the channels were split, for all successful trials, into four time windows (TSD, D1, DSD and D2) which correspond to the time windows in the delayed saccade task. The wideband signals were bandpass-filtered between $20 \mathrm{~Hz}$ and $60 \mathrm{~Hz}$ to extract the LFPs [8].

Next, we studied the emergence of phase-synchronization patterns among the 8 brain subregions for the successful trials. The goal here was the identification of the time windows in which chimera-like states might spontaneously emerge. To this end, we extracted the instantaneous phase of each channel for all successful trials employing the Hilbert transform in Matlab and calculated the average order parameter $\rho$ [10] over the channels in each brain subregion for the 4 time windows of interest (namely, TSD, D1, DSD and D2), for all successful trials. At the end, we calculated the chimeralike $\chi$ and metastability $\lambda$ indices, and coalition entropy $H_{c}$ [9] for each time window and successful trial.

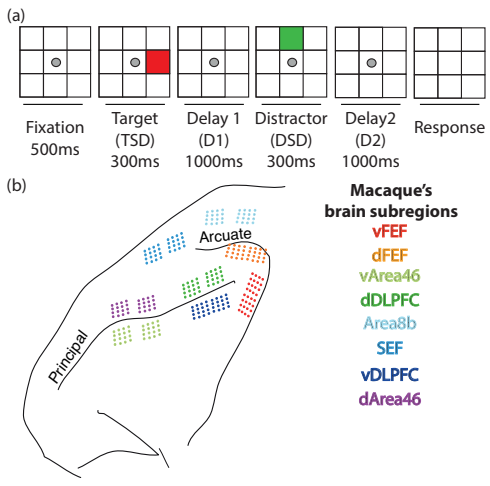

Fig. 1. Experimental setup. Panel (a): The time windows in the trials and their durations in ms. Panel (b): The locations of implanted electrodes in the macaque's brain and their labels (Macaque's brain subregions column). Both panels were recreated from [7].

\section{A. The Hilbert Transform}

A traditional approach for the computation of the instantaneous phase of a signal is the use of the Hilbert transform (HT). The HT $\hat{y}(t)$ of a signal $y(t)$ can be calculated by

$$
\hat{y}(t)=\frac{1}{\pi} \int_{-\infty}^{\infty} \frac{y(\tau)}{t-\tau} \mathrm{d} \tau .
$$

Combining the initial signal $y(t)$ and the output of the HT $\hat{y}(t)$, one gets the analytic representation of the signal

$$
Y(t)=y(t)+i \hat{y}(t)
$$

where $i^{2}=-1$. The analytic signal is a complex number and can be expressed as

$$
Y(t)=A(t) e^{i \phi(t)},
$$

where $A(t)$ is the amplitude and $\phi(t)$ the instantaneous phase of the signal at time $t$. We use the instantaneous phase $\phi(t)$ to compute $\rho$ [10], the chimera-like $\chi$ and metastability $\lambda$ indices, and coalition entropy $H_{c}$ [9].

\section{B. Order parameter $\rho$}

The order parameter $\rho$ quantifies the synchronization among the 8 subregions in the macaque's brain (see fig. 1(b)). Particularly, it can be calculated by

$$
\rho(t)=\frac{1}{N} \sum_{j=1}^{N} e^{i \phi_{j}(t)},
$$

where $\phi_{j}(t)$ is the instantaneous phase of channel $j$ and $N$ the number of channels considered. In our case, each brain 
subregion comprises $N=32$ channels. According to Eq. (4), $\rho$ takes values in $[0,1]$, whereby $\rho=1$ corresponds to complete synchronization and $\rho=0$ to complete desynchronization.

\section{Metastability index $\lambda$}

Following [9], $\lambda$ quantifies the level of metastability in the system. By metastability, we mean how much phase-synchrony varies in each brain subregion over time, and can be calculated by

$$
\lambda=\left\langle\sigma_{m e t}\right\rangle_{C_{m}},
$$

where

$$
\sigma_{m e t}(m)=\frac{1}{T-1} \sum_{t=1}^{T}\left(\rho_{m}(t)-\left\langle\rho_{m}\right\rangle_{T}\right)^{2}
$$

and $T$ is the time length of the data. In Eq. (5), $C_{m}$ is the set of all 8 brain subregions and $m=1,2, \ldots, 8$. For a given subregion, the variance $\sigma_{m e t}(m)$ of $\rho_{m}(t)$ over all time steps, quantifies the variation in time of the synchrony in the subregion. Averaging over all 8 brain subregions in Eq. (5), we obtain $\lambda$, an index of the metastability in the data from the macaque's brain subregions. In our study, we normalize $\lambda$ in $[0,1]$ to compare with $\chi$.

\section{Chimera-like index $\chi$}

Following again [9], $\chi$ quantifies how chimera-like the data are in time, meaning how much phase-synchrony varies over the brain subregions at each time $t$. It can be calculated

$$
\chi=\left\langle\sigma_{c h i}\right\rangle_{T}
$$

where,

$$
\sigma_{c h i}(t)=\frac{1}{M-1} \sum_{m=1}^{M}\left(\rho_{m}(t)-\left\langle\rho_{m}\right\rangle_{M}\right)^{2}
$$

and $M=8$ is the number of brain subregions. In Eq. (8), $\sigma_{c h i}(t)$ is an instantaneous quantity that gives the variance of $\rho_{m}(t)$ over all $M=8$ brain subregions at time $t$. Taking the average of $\sigma_{c h i}(t)$ in time, indicates how chimera-like the data are in time. In our study, we normalize $\chi$ in $[0,1]$ to compare with the normalized $\lambda$. In the next, we use the normalized $\chi$ and $\lambda$ values in $[0,1]$ to compare.

\section{E. Coalition entropy $H_{c}$}

$H_{c}$ is the coalition entropy of the system and quantifies how repeatedly the system visits the same metastable chimera state or has a large variety of metastable chimera states it is visiting [9]. It is defined by

$$
H_{c}=-\frac{1}{\log _{2}|S|} \sum_{s \in S} p(s) \log _{2}(p(s)),
$$

where $S$ is the set of distinct coalitions generated by the system and $p(s)$ the probability of coalition $s$ to arise at any given time $t$. We define a coalition $s$ to arise at time $t$ if $\rho_{c}(t)>\gamma$ for all $c \in s$, where $\gamma=0.8$ is a synchronization threshold. $H_{c}$ is normalized to the interval $[0,1]$. If all possible coalitions are equiprobable, $H_{c}$ is maximized (i.e. $H_{c}=1$ ). In contrast, if the system resides permanently in the same state which means that only one coalition arises, then $H_{c}=0$.

\section{RESUlTS}

In fig. 2, we show the mean and standard errors of the normalized $\chi$ values over the 240 trials for the time windows TSD, D1, DSD and D2. The biggest average $\chi$ appears for windows D1 and D2. On the other hand, TSD and DSD have the lowest average chimera-like indices. In the transition from TSD to D1 and from DSD to D2, these averages tend to increase. We also run paired $t$-tests for the time windows to study the statistical significance over the 240 successful trials and revealed that the average normalized $\chi$ were significantly higher for these two transitions, especially for the one from DSD to D2. This signifies their statistical significance and thus, cannot be attributed to randomness.

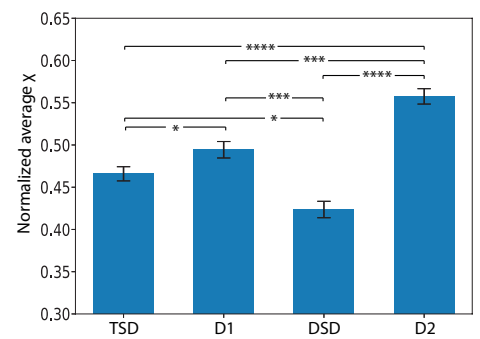

Fig. 2. The mean values and standard errors of the normalized average $\chi$ among all trials for time windows TSD, D1, DSD and D2 (with $p<0.05$ $(*) ; p<0.005$ (**); $p<5 \times 10^{-5}(* * *) ; p<5 \times 10^{-10}$ (****)). Note that the horizontal lines with the stars denote the pairs used in the $t$-tests along with their corresponding $p$-values for their statistical significance.

Similarly, fig. 3 shows that the highest averaged normalized metastability indices are attributed to the TSD and DSD time windows. In contrast, D1 and D2 have the lowest averaged metastability indices. Here, we see a decreasing trend for the averages $\lambda$ for the transitions from TSD to D1 and from DSD to D2. The paired $t$-tests for the time windows also revealed that these indices were significantly low for both transitions and signify their statistical significance, meaning they cannot be attributed to randomness.

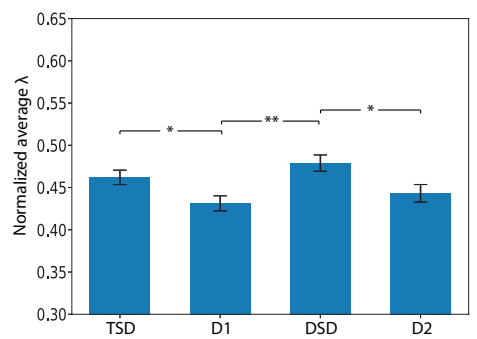

Fig. 3. The mean values and standard errors of the normalized average $\lambda$ among all trials for time windows TSD, D1, DSD and D2 (with $p<0.05$ $(*) ; p<0.005$ (**); $p<5 \times 10^{-5}$ (***); $p<5 \times 10^{-10}$ (****)). Note that the horizontal lines with the stars denote the pairs used in the $t$-tests along with their corresponding $p$-values for their statistical significance.

In agreement with figs. 2 and 3, fig. 4 shows that the highest averaged $H_{c}$ appears again for D1 and D2. On the contrary, the lowest values appear for the TSD and DSD time windows. The paired $t$-tests also revealed that the $H_{c}$ values are significantly higher in D1 and D2 and cannot be attributed to randomness. 
Low $H_{c}$ signifies that the system resides closer to the same state whether fully synchronized, fully desynchronized, or any sort of chimera-like state, in contrast to a higher value which shows the system repeatedly visits a larger repertoire of metastable chimera-like states.

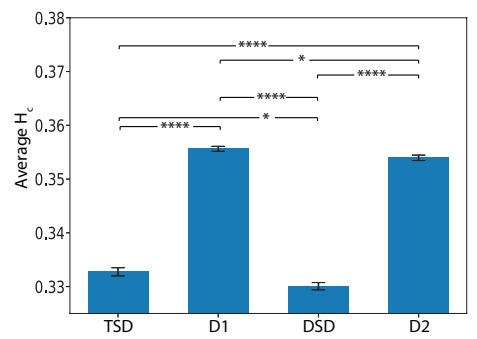

Fig. 4. The means and standard errors of the average $H_{c}$ among all trials for all four time windows TSD, D1, DSD and D2 (with $p<0.05(*) ; p<$ $\left.0.005(* *) ; p<5 \times 10^{-5}(* * *) ; p<5 \times 10^{-10}(* * * *)\right)$. Note that the horizontal lines with the stars denote the pairs used in the $t$-tests along with their corresponding $p$-values for their statistical significance.

The results in figs. 2, 3 and 4 imply that time windows D1 and D2 are characterized by weakly metastable, chimera-like states, whereas TSD and DSD, mainly by metastable states. In D1 and D2, the system repeatedly visits a larger repertoire of weakly metastable chimera-like states whereas in TSD and DSD, it resides closer to the same metastable state.

Finally, fig. 5(a) shows a snapshot of the 256 phases grouped by their corresponding brain subregions, where coherent and decoherent subregions coexist. Particularly, channels in subregions 1, 2, 3, 6 and 8 have their phases synchronized (coherent group) whereas channels in groups 4,5 and 7 have their phases desynchronized (decoherent group). The coexistence of a spatio-temporal coherent and decoherent group of channels is the manifestation of chimera-like behavior. For comparison, panel (b) shows a snapshot where the phases are all desynchronized.
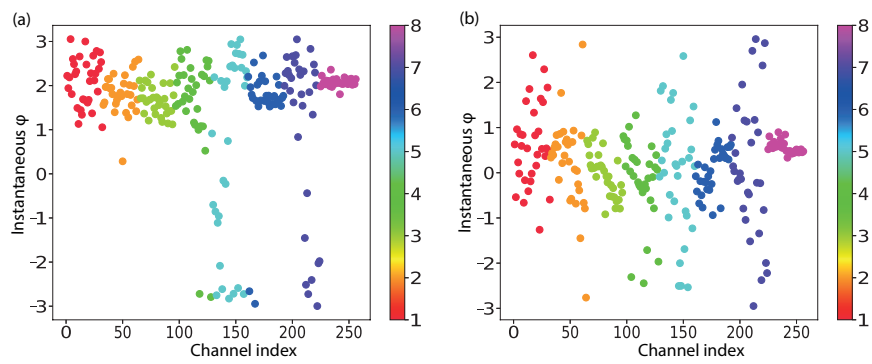

Fig. 5. Panel (a): A snapshot of instantaneous phases $\phi$ showing coexistence of coherent and decoherent groups of phases in the macaque's brain subregions. Panel (b): A snapshot of desynchronized instantaneous phases $\phi$ in the various macaque's brain subregions. Note that in both panels, the horizontal axis is the index of the channel and the vertical the instantaneous phases $\phi$ (in radians). The color-codes on the right represent the 8 groups of channels mapped according to the 8 brain subregions in fig. 1(b).

\section{Discussion}

In this work, we sought to study the emergence of chimeralike states in the macaque brain composed of cortical subregions while the animal was performing a delayed saccade task many times. To do so, we used the chimera-like and metastability indices, and coalition entropy for the successful trials and split the data in 4 experimentally relevant time windows.

Our results show that time windows D1 and D2 are characterized by weakly metastable chimera-like states, whereas TSD and DSD mainly by metastable states. In D1 and D2, the system repeatedly visits a larger repertoire of weakly metastable chimera-like states whereas in TSD and DSD, it resides closer to the same metastable state.

Our work shows the presence of chimera-like states in the recorded data from the macaques' brain in D1 and D2 time windows. We observed the emergence of weakly metastable chimera-like states after the presentation of the stimulus in the screen with the metastability index to decrease to lower values after the stimulus. Our findings suggest that metastable chimera-like states emerge in the delay periods D1 and D2, after the display of the stimuli.

\section{REFERENCES}

[1] Taylor, D. M., Tillery, S. I. H., \& Schwartz, A. B. (2002). Direct cortical control of 3D neuroprosthetic devices. Science, 296(5574), 1829-1832

[2] Li, J., \& Cichocki, A. (2014). Deep learning of multifractal attributes from motor imagery induced EEG. In International Conference on Neural Information Processing (pp. 503-510). Springer, Cham.

[3] McFarland, D. J., Sarnacki, W. A., \& Wolpaw, J. R. (2010). Electroencephalographic (EEG) control of three-dimensional movement. Journal of Neural Engineering, 7(3), 036007

[4] Felton, E. A., Wilson, J. A., Williams, J. C., \& Garell, P. C. (2007). Electrocorticographically controlled brain-computer interfaces using motor and sensory imagery in patients with temporary subdural electrode implants: report of four cases. Journal of Neurosurgery, 106(3), 495-500

[5] Flint, R. D., Wright, Z. A., Scheid, M. R., \& Slutzky, M. W. (2013). Long term, stable brain machine interface performance using local field potentials and multiunit spikes. Journal of Neural Engineering, 10(5), 056005

[6] Li, J., Wang, Y., Zhang, L., \& Jung, T. P. (2012). Combining ERPs and EEG spectral features for decoding intended movement direction. In Engineering in Medicine and Biology Society, 2012 Annual International Conference of the IEEE (pp. 1769-1772). IEEE

[7] Parthasarathy, A., Herikstad, R., Bong, J. H., Medina, F. S., Libedinsky, C., \& Yen, S. C. (2017). Mixed selectivity morphs population codes in prefrontal cortex. Nature Neuroscience, 20(12), 1770-1779

[8] Gregoriou, G. G., Gotts, S. J., Zhou, H., \& Desimone, R. (2009). Highfrequency, long-range coupling between prefrontal and visual cortex during attention. Science, 324(5931), 1207-1210

[9] Shanahan, M. (2010). Metastable chimera states in community-structured oscillator networks. Chaos, 20(1), 013108

[10] Kuramoto, Y., \& Battogtokh, D. (2002). Coexistence of coherence and incoherence in non locally coupled phase oscillators. Nonlin. Phen. in Complex Sys., 5, 380-385

[11] Abrams, D. M., \& Strogatz, S. H. (2004). Chimera states for coupled oscillators. Phys. Rev. Lett., 93, 174102

[12] Gómez-Gardeñes, J., Zamora-López, G., Moreno, Y., \& Arenas, A (2010). From modular to centralized organization of synchronization in functional areas of the cat cerebral cortex. PLOS One, 5(8), e12313

[13] Hizanidis, J., Kouvaris, N. E., Zamora-López, G., Díaz-Guilera, A., \& Antonopoulos, C. G. (2016). Chimera-like states in modular neural networks. Scientific Reports, 6, 19845

[14] Santos, M. S., Szezech, J. D., Borges, F. S., Iarosz, K. C., Caldas, I. L., Batista, A. M., Viana, R. L. \& Kurths, J. (2017). Chimera-like states in a neuronal network model of the cat brain. Chaos, Solitons \& Fractals, 101, 86-91

[15] Chouzouris, T., Omelchenko, I., Zakharova, A., Hlinka, J., Jiruska, P., \& Schöll, E. (2017). Chimera states in brain networks: Empirical neural vs. modular fractal connectivity. Chaos, 28, 10.1063/1.5009812 